MATHEMATICS OF COMPUTATION

Volume 72, Number 244, Pages 1817-1839

S 0025-5718(03)01485-6

Article electronically published on January 8, 2003

\title{
ANALYSIS OF TRIANGLE QUALITY MEASURES
}

\author{
PHILIPPE P. PÉBAY AND TIMOTHY J. BAKER
}

\begin{abstract}
Several of the more commonly used triangle quality measures are analyzed and compared. Proofs are provided to verify that they do exhibit the expected extremal properties. The asymptotic behavior of these measures is investigated and a number of useful results are derived. It is shown that some of the quality measures are equivalent, in the sense of displaying the same extremal and asymptotic behavior, and that it is therefore possible to achieve a concise classification of triangle quality measures.
\end{abstract}

\section{INTRODUCTION}

Assessment of mesh quality is an important requirement in both the selection of a finite element mesh and the evaluation of meshes that have undergone adaptation [2] 5. Several measures of element quality have been proposed [1, 8, 11 based on the dimensionless ratios of various geometric parameters. Apart from the work of [11, there appears to be almost no discussion in the literature on the relative merits of these particular quality measures. More recently, alternative quality measures have been suggested [3, 7, 9, 10]. These alternative measures are derived from the singular values of a matrix whose columns are formed by the edge vectors of the mesh element.

An element is said to be degenerate if its volume is zero. Let $Q$ be a quality measure defined for any nondegenerate simplex $t$ and let the range of $Q$ be the real interval $[1,+\infty[$. It is assumed that $Q$ satisfies the following extremal properties:

(i) $Q$ attains its minimum value of 1 if and only if $t$ is a regular simplex;

(ii) $Q$ has no other extrema.

In many cases, these extremal properties have been assumed, or stated, without proof. Although the extremal behavior of these quality measures might appear obvious, we believe that this behavior should be established rigorously and precise bounds should be found.

In this paper we examine the triangular case since properties of the triangle are particularly amenable to analysis. We consider several of the more commonly used triangle quality measures, provide proofs of their extremal properties, and examine their asymptotic behavior. Our goal is to provide a number of useful results on triangle quality measures that may lead to a better assessment of both planar triangulations and triangulated surfaces.

Received by the editor July 9, 2001 and, in revised form, December 21, 2001.

2000 Mathematics Subject Classification. Primary 32B25, 65M50; Secondary 51N20.

Key words and phrases. Triangulation, surface triangulation, triangle quality, mesh quality.

The first author was supported in part by CNRS, UMR 5585, France. 


\section{Preliminaries}

In this paper, we consider a nondegenerate triangle $t=A B C$ with area $\mathcal{A}$, half-perimeter $p$, edges of lengths $a=B C, b=A C$ and $c=A B$, and denote the angle at vertex $A$ (resp. $B, C$ ) as $\alpha$ (resp. $\beta, \gamma)$ and the radius of the inscribed (resp. circumscribed) circle of $t$ as $r$ (resp. $R$ ). In addition, the vertices $A, B$ and $C$ are defined respectively by the position vectors $\mathbf{v}_{0}, \mathbf{v}_{1}$ and $\mathbf{v}_{2}$ in an arbitrary orthonormal affine reference frame. For simplicity, we choose a frame of reference parallel to the plane of the triangle $t$, in which case the coordinates of the position vectors $\mathbf{v}_{0}, \mathbf{v}_{1}$ and $\mathbf{v}_{2}$ are denoted respectively as $\left(x_{0}, y_{0}\right),\left(x_{1}, y_{1}\right)$ and $\left(x_{2}, y_{2}\right)$.

We shall also use the following standard norm-like notations:

$$
\begin{aligned}
|t|_{0} & =\min (a, b, c), \\
|t|_{2} & =\sqrt{a^{2}+b^{2}+c^{2}} \\
|t|_{\infty} & =\max (a, b, c), \\
\theta_{0} & =\min (\alpha, \beta, \gamma), \\
\theta_{\infty} & =\max (\alpha, \beta, \gamma) .
\end{aligned}
$$

We shall assume without proof a number of results from elementary geometry (see, e.g., 6] for proofs and details). In particular, we will make use of the well-known relations

$$
2 R=\frac{a b c}{2 \mathcal{A}}=\frac{a}{\sin \alpha}=\frac{b}{\sin \beta}=\frac{c}{\sin \gamma},
$$

where $\mathcal{A}$ is given by

$$
\mathcal{A}=r p
$$

as well as by Heron's formula

$$
\mathcal{A}=\sqrt{p(p-a)(p-b)(p-c)} .
$$

We also recall two important results, valid for any $n \in \mathbb{N}^{*}$ : the arithmetic-geometric inequality

$$
\left(\forall\left(u_{1}, \ldots, u_{n}\right) \in \mathbb{R}_{+}^{n}\right) \quad \sqrt[n]{\prod_{k=1}^{k=n} u_{k}} \leq \frac{1}{n} \sum_{k=1}^{k=n} u_{k} ;
$$

and the Cauchy-Schwarz inequality

$$
\left(\forall\left(u_{1}, \ldots, u_{n}\right) \in \mathbb{R}_{+}^{n}\right) \quad \sum_{k=1}^{k=n} u_{k} \leq \sqrt{n \sum_{k=1}^{k=n} u_{k}^{2}} .
$$

We shall use the terms needle and flattened triangle to refer to two types of nearly degenerate triangles. More precisely, these are defined as follows.

Definition 1.1. A needle is a nondegenerate triangle that has one and only one angle close to 0 .

Definition 1.2. A flattened triangle is a nondegenerate triangle that has one angle close to $\pi$. 


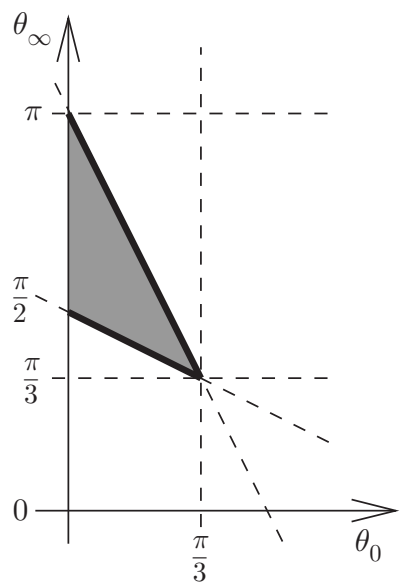

Figure 1 . Gray: $\Omega$, definition set of $\theta_{0}$ and $\theta_{\infty}$; the thin edge with its endpoints is excluded.

\section{Extremal ANGLES}

One of the most commonly accepted means of measuring triangle quality is to examine $\theta_{0}$ or $\theta_{\infty}$. By definition of these angles, one always has

$$
0<\theta_{0} \leq \frac{\pi}{3} \leq \theta_{\infty}
$$

Moreover, the implicit condition

$$
\theta_{0} \leq \pi-\theta_{0}-\theta_{\infty} \leq \theta_{\infty}
$$

is equivalent to

$$
\theta_{\infty} \leq \pi-2 \theta_{0}, \quad 2 \theta_{\infty} \geq \pi-\theta_{0} .
$$

For the sake of clarity, (2.1) and (2.3) are summarized by denoting as $\Omega$ the corresponding definition set of $\left(\theta_{0}, \theta_{\infty}\right)$, shown in Figure 1 . The bold edges correspond to the two different kinds of isosceles triangle: acute on the lower one, obtuse on the upper one, which meet when $\theta_{0}=\theta_{\infty}=\frac{\pi}{3}$ (i.e., the equilateral case).

In other words, (2.3) gives the bounds for the maximal angle depending on the minimal one

$$
\left.\left.\left(\forall \theta_{0} \in\right] 0, \frac{\pi}{3}\right]\right) \quad \frac{\pi}{2}-\frac{\theta_{0}}{2} \leq \theta_{\infty} \leq \pi-2 \theta_{0},
$$

while 2.1) and (2.2) conversely provide

$$
\begin{aligned}
& \left(\forall \theta _ { \infty } \in \left[\frac{\pi}{3}, \frac{\pi}{2}[) \quad \pi-2 \theta_{\infty} \leq \theta_{0} \leq \frac{\pi}{2}-\frac{\theta_{\infty}}{2},\right.\right. \\
& \left(\forall \theta _ { \infty } \in \left[\frac{\pi}{2}, \pi[) \quad 0<\theta_{0} \leq \frac{\pi}{2}-\frac{\theta_{\infty}}{2} .\right.\right.
\end{aligned}
$$

Two useful inequalities arise directly: from (2.4),

$$
\left(\forall\left(\theta_{0}, \theta_{\infty}\right) \in \Omega\right) \quad \frac{\pi}{2} \leq \frac{\theta_{0}}{2}+\theta_{\infty} \leq \pi-\frac{3 \theta_{0}}{2} ;
$$

and from (2.5) and (2.6),

$$
\left(\forall\left(\theta_{0}, \theta_{\infty}\right) \in \Omega\right) \quad 0<\max \left(\frac{\theta_{\infty}}{2}, \pi-\frac{3 \theta_{\infty}}{2}\right) \leq \frac{\theta_{\infty}}{2}+\theta_{0} \leq \frac{\pi}{2} .
$$


These inequalities may be rewritten strictly over the interior $\stackrel{\circ}{\Omega}$ of $\Omega$ as

$$
\left(\forall\left(\theta_{0}, \theta_{\infty}\right) \in \stackrel{\circ}{\Omega}\right) \quad \frac{\pi}{2}<\frac{\theta_{0}}{2}+\theta_{\infty}<\pi-\frac{3 \theta_{0}}{2}
$$

and

$$
\left(\forall\left(\theta_{0}, \theta_{\infty}\right) \in \stackrel{\circ}{\Omega}\right) \quad 0<\max \left(\frac{\theta_{\infty}}{2}, \pi-\frac{3 \theta_{\infty}}{2}\right)<\frac{\theta_{\infty}}{2}+\theta_{0}<\frac{\pi}{2} .
$$

Before examining how the more commonly used nondimensional quality measures are related to these extremal angles, it is useful to prove the following result. It seems obvious but we have not observed it in the literature.

Lemma 2.1. The three angles of a nondegenerate triangle are sorted in the same order as the lengths of their respective opposite edges.

Proof. We can assume, without loss of generality, that $a \leq b \leq c$. Hence, it can be directly deduced from (1.1) that $\sin \alpha \leq \sin \beta \leq \sin \gamma$. Now, both $\alpha$ and $\beta$ are necessarily smaller than $\frac{\pi}{2}$ and the sine function is monotonically increasing over ] $\left.0, \frac{\pi}{2}\right]$, thus $\alpha \leq \beta$. Concerning $\gamma$, two cases may occur: if it is acute, then the same argument can be used; if it is obtuse or right, then clearly $\beta \leq \gamma$. Therefore, in either case, we have $\alpha \leq \beta \leq \gamma$.

Remark 2.2. In other words, the length of the edge opposite angle $\theta_{0}$ (resp. $\theta_{\infty}$ ) is $|t|_{0}\left(\right.$ resp. $\left.|t|_{\infty}\right)$.

\section{RADIUS-RATIO}

A convenient, nondimensional and thus homogeneous, quality measure consists in comparing $r$ and $R$, resulting in the radius-ratio, defined as

$$
\rho=\frac{R}{r} \text {. }
$$

Combining (1.1) with (1.2) leads to

$$
a b c=4 R r p,
$$

and then a further application of (1.1) gives the following expressions for $\rho$ in terms of the angles of a triangle:

$$
\rho=\frac{\sin \alpha+\sin \beta+\sin \gamma}{2 \sin \alpha \sin \beta \sin \gamma}
$$

whence, since $\alpha+\beta+\gamma=\pi$,

$$
\rho=\frac{\sin \alpha+\sin \beta+\sin (\alpha+\beta)}{2 \sin \alpha \sin \beta \sin (\alpha+\beta)},
$$

or, using the extremal angles,

$$
\rho=\frac{\sin \theta_{0}+\sin \theta_{\infty}+\sin \left(\theta_{0}+\theta_{\infty}\right)}{2 \sin \theta_{0} \sin \theta_{\infty} \sin \left(\theta_{0}+\theta_{\infty}\right)} .
$$

If we write $p=\tan \frac{\alpha}{2}$ and $q=\tan \frac{\beta}{2}$ in (3.4), then

$$
\rho=\frac{\left(1+p^{2}\right)\left(1+q^{2}\right)}{4 p q(1-p q)} .
$$


3.1. Extremum. The mapping $x \mapsto \tan \frac{x}{2}$ is a $\mathcal{C}^{\infty}$ function, bijective from $] 0, \pi[$ onto $\mathbb{R}_{+}^{*}$. Thus both parametrizations of $\rho$ are $\mathcal{C}^{\infty}$-equivalent and, hence, differential properties are bijectively transported from one to the other. Now, it is easy to prove that the condition

$$
(\alpha, \beta) \in] 0, \pi\left[^{2}, \quad \alpha+\beta<\pi,\right.
$$

is equivalent to

$$
(p, q) \in \mathbb{R}_{+}^{* 2}, \quad p q<1 .
$$

Therefore, let us consider the mapping

$$
\begin{array}{cccc}
f: \quad \mathcal{D} & \longrightarrow & \mathbb{R}_{+}^{*}, \\
(p, q) & \longmapsto & \frac{\left(1+p^{2}\right)\left(1+q^{2}\right)}{p q(1-p q)},
\end{array}
$$

where $\mathcal{D}$ is the domain 1 defined by condition (3.8). Clearly, $f$ is $\mathcal{C}^{\infty}$, when $\mathcal{D}$ is open; hence, any extremum of $f$ is attained at a stationary point. We have

$$
\begin{aligned}
& \frac{\partial f}{\partial p}(p, q)=\frac{\left(1+q^{2}\right)\left(p^{2}+2 q p-1\right)}{q p^{2}(1-p q)^{2}}, \\
& \frac{\partial f}{\partial q}(p, q)=\frac{\left(1+p^{2}\right)\left(q^{2}+2 p q-1\right)}{p q^{2}(1-p q)^{2}},
\end{aligned}
$$

and $(p, q)$ is a stationary point if and only if

$$
p^{2}+2 q p-1=q^{2}+2 p q-1=0 .
$$

Hence, this implies $p=q$ and, since we know that the variables substitution is bijective, $\alpha=\beta$. Now, assuming this necessary condition is satisfied, (3.12) becomes $3 p^{2}=1$, so that $p=q=\frac{1}{\sqrt{3}}$ since these values must be positive. In other words, the only critical point of $\rho$ is met when $\alpha=\beta=\frac{\pi}{3}$ (i.e., for an equilateral triangle). In order to check whether this case corresponds, as expected, to a minimum, one has to make sure that the hessian matrix is positive definite. The second-order derivatives are given by

$$
\begin{aligned}
\frac{\partial^{2} f}{\partial p^{2}}(p, q) & =2 \frac{\left(1+q^{2}\right)\left(p^{3} q+3 p^{2} q^{2}-3 p q+1\right)}{q p^{3}(1-p q)^{3}}, \\
\frac{\partial^{2} f}{\partial q^{2}}(p, q) & =2 \frac{\left(1+p^{2}\right)\left(q^{3} p+3 p^{2} q^{2}-3 p q+1\right)}{p q^{3}(1-p q)^{3}}, \\
\frac{\partial^{2} f}{\partial p \partial q}(p, q) & =\frac{p q+5}{(1-p q)^{3}}+\frac{\left(p^{2}+q^{2}-1\right)(3 p q-1)}{p^{2} q^{2}(1-p q)^{3}}
\end{aligned}
$$

which gives, when $p=q=\frac{1}{\sqrt{3}}$,

$$
\begin{aligned}
\frac{\partial^{2} f}{\partial p^{2}}\left(\frac{1}{\sqrt{3}}, \frac{1}{\sqrt{3}}\right) & =\frac{\partial^{2} f}{\partial q^{2}}\left(\frac{1}{\sqrt{3}}, \frac{1}{\sqrt{3}}\right)=36, \\
\frac{\partial^{2} f}{\partial p \partial q}\left(\frac{1}{\sqrt{3}}, \frac{1}{\sqrt{3}}\right) & =18 .
\end{aligned}
$$

Thus the hessian determinant is equal to $36^{2}-18^{2}>0$ and the first diagonal entry is $36>0$. Hence, the hessian matrix is locally positive definite around the critical point, which therefore corresponds to a strict minimum of $f$. It follows from the

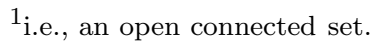


$\mathcal{C}^{\infty}$-equivalent parametrizations, that $\rho$ is minimal only for an equilateral triangle, since $\arctan \frac{1}{\sqrt{3}}=\frac{\pi}{3}$. In this case, the radius-ratio is

$$
\frac{1}{4} f\left(\frac{1}{\sqrt{3}}, \frac{1}{\sqrt{3}}\right)=\left(\frac{4}{3}\right)^{2} \times \frac{3^{2}}{2}=2 .
$$

3.2. Asymptotic behavior. An obvious link between the radius-ratio and the extremal angles can be deduced from (3.5):

$$
\rho=\frac{\sin \theta_{0}+\sin \theta_{\infty}+\sin \left(\theta_{0}+\theta_{\infty}\right)}{2 \sin \theta_{0} \sin \theta_{\infty} \sin \left(\theta_{0}+\theta_{\infty}\right)} .
$$

It is therefore interesting to examine how sensitive the radius-ratio is to the information provided by an extremal angle measurement. In particular, it is well known (cf. [12]) that among all triangulations of the convex hull of a given set of points in $\mathbb{R}^{2}$, any Delaunay triangulation maximizes the minimal vertex angle; this property is generally considered to be a guarantee for the quality of the elements obtained through a Delaunay based algorithm, while (3.19) shows immediately that this alternative view of triangle quality is not bijectively linked to the radius-ratio.

Figure 2 shows parts of the surface defined by the dependence of $\frac{\rho}{2}$ on two angles of the triangle. The radius-ratio is normalized for convenience, since most
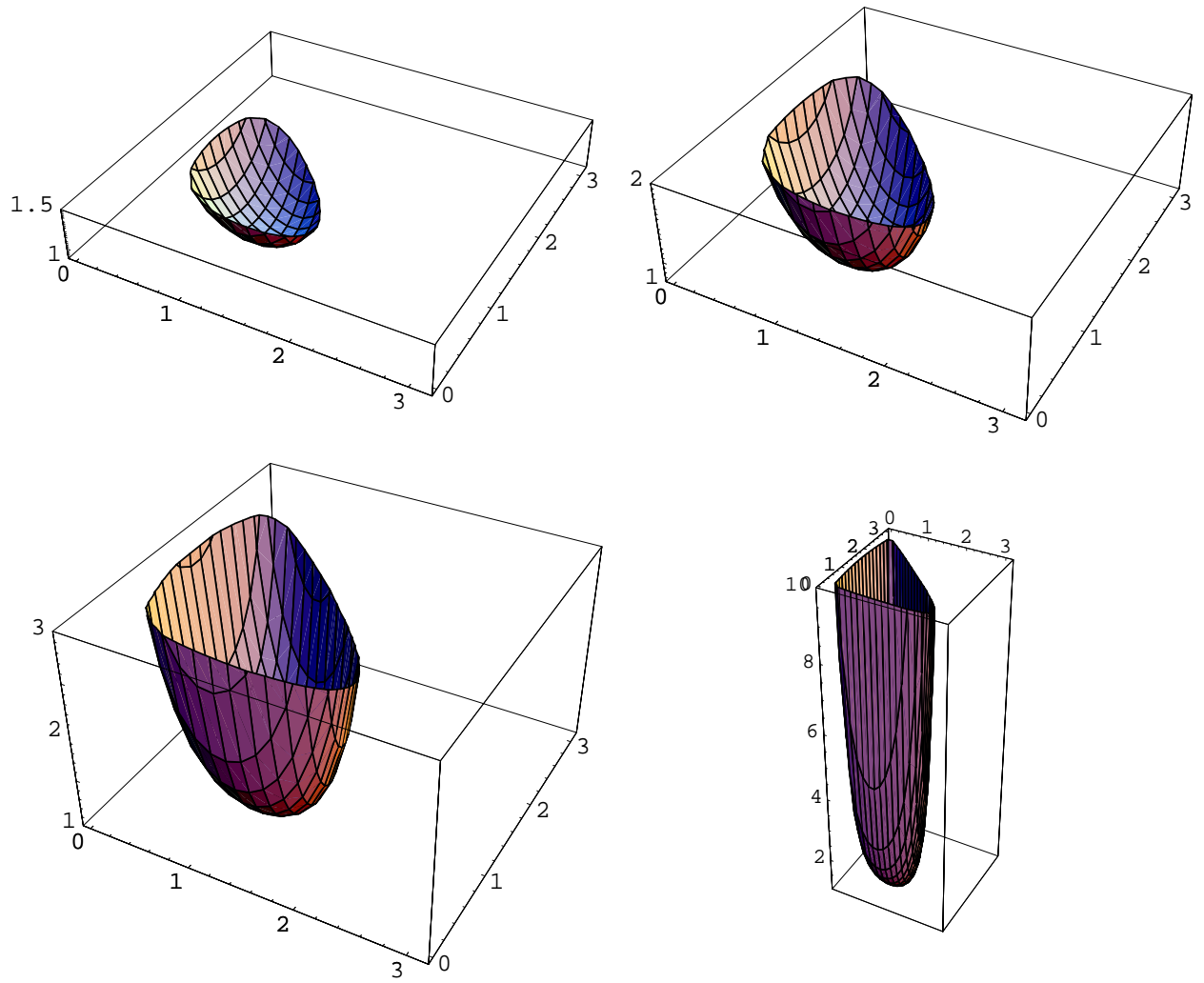

FIgURE 2. Surface $z=\frac{\rho}{2}$ as a function of two angles in radians: (i) $z<1.5$; (ii) $z<2$; (iii) $z<3$; (iv) $z<10$. 


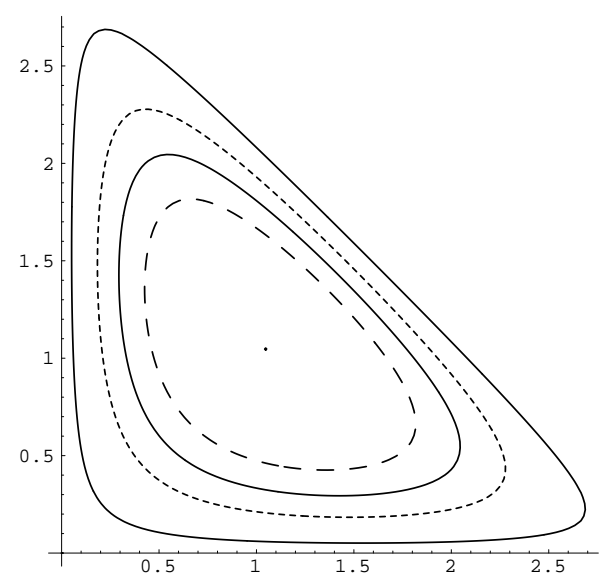

Figure 3. Isovalues of $\frac{\rho}{2}$ as a function of two angles in radians from the center to the exterior: 1 (spot), 1.5 (dashed), 2, 3 (dotted), 10 .

users tend to prefer that the quality of an equilateral triangle is 1; Figure 3 shows the corresponding isovalues.

Figures 2 and 3 clearly indicate a weak dependence between $\rho$ and the extremal angles. Nevertheless, it is possible to derive some results, providing bounds for the radius-ratio measure for given extremal angular values. More precisely, (3.5) gives

$$
\begin{aligned}
\frac{\partial \rho}{\partial \theta_{0}}\left(\theta_{0}, \theta_{\infty}\right) & =\frac{-\cos \left(\frac{\theta_{\infty}}{2}+\theta_{0}\right)}{8 \sin ^{2} \frac{\theta_{0}}{2} \sin \frac{\theta_{\infty}}{2} \cos \frac{\theta_{0}+\theta_{\infty}}{2}}, \\
\frac{\partial \rho}{\partial \theta_{\infty}}\left(\theta_{0}, \theta_{\infty}\right) & =\frac{-\cos \left(\frac{\theta_{0}}{2}+\theta_{\infty}\right)}{8 \sin \frac{\theta_{0}}{2} \sin ^{2} \frac{\theta_{\infty}}{2} \cos \frac{\theta_{0}+\theta_{\infty}}{2}} .
\end{aligned}
$$

It is now straightforward to study the variations of $\rho$ when the minimal angle is fixed: for any given $\theta_{0}$ in $\left.] 0, \frac{\pi}{3}\right]$, it follows from (3.21) and (2.9) that both the denominator and the numerator of $\frac{\partial \rho}{\partial \theta_{\infty}}$ are strictly positive for $\theta_{\infty}$ in the interval ]$\frac{\pi}{2}-\frac{\theta_{0}}{2}, \pi-2 \theta_{0}\left[\right.$, since $0<\theta_{0} \leq \frac{\pi}{3}$. Combining with (2.4), it then follows that $\theta_{\infty} \mapsto \rho\left(\theta_{0}, \theta_{\infty}\right)$ is a continuous strictly monotonically increasing function over the closed interval $\left[\frac{\pi}{2}-\frac{\theta_{0}}{2}, \pi-2 \theta_{0}\right]$ and therefore attains a unique minimum (resp. maximum) at the lower (resp. upper) bound of this interval. In other words,

\section{Proposition 3.1.}

$$
\begin{array}{ll}
\left.\left.\left(\forall \theta_{0} \in\right] 0, \frac{\pi}{3}\right]\right) \quad & \inf _{\theta_{\infty}} \rho=\frac{1+\sin \frac{\theta_{0}}{2}}{\sin \theta_{0} \cos \frac{\theta_{0}}{2}}, \\
\left.\left.\left(\forall \theta_{0} \in\right] 0, \frac{\pi}{3}\right]\right) & \sup _{\theta_{\infty}} \rho=\frac{1+\cos \theta_{0}}{\sin \theta_{0} \sin 2 \theta_{0}} .
\end{array}
$$

Example 3.2. Let the minimal angle be equal to $10^{\circ}$ (resp. $20^{\circ}$ ), then the normalized radius-ratio ranges from circa 3.14 to 16.7 (resp. 1.74 to 4.41). Hence, even for "reasonable" values of $\theta_{0}$, it is clear that the radius-ratio is only weakly dependent on the minimal angle. 

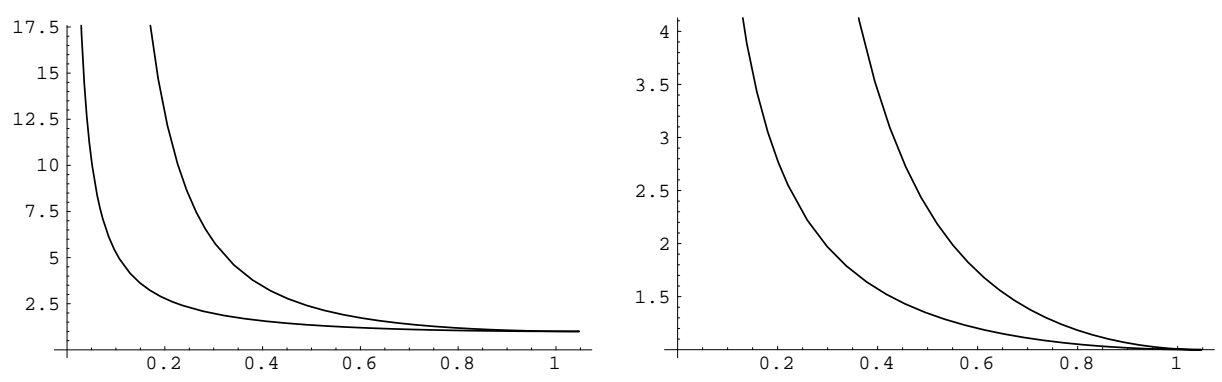

Figure 4. Graphs at two different scales providing the range of $\frac{\rho}{2}$ as a function of the minimal angle in radians.

Conversely, if the maximal angle is fixed, the sign of $\frac{\partial \rho}{\partial \theta_{0}}$ is, according to (3.21) and (2.10), strictly negative. Hence, it follows from (2.5) and (2.6) that for any given $\theta_{\infty}$ in $\left[\frac{\pi}{3}, \pi\right.$ [ the mapping $\theta_{0} \mapsto \rho\left(\theta_{0}, \theta_{\infty}\right)$ is strictly monotonically decreasing over $\left.] \max \left(0, \pi-2 \theta_{\infty}\right), \frac{\pi}{2}-\frac{\theta_{\infty}}{2}\right]$. Here, the two subcases, depending on the lower bound of the interval, have to be discussed. First, if $\frac{\pi}{3} \leq \theta_{\infty}<\frac{\pi}{2}$, from the definition interval of $\theta_{0}$ and the fact that $\theta_{0} \mapsto \rho\left(\theta_{0}, \theta_{\infty}\right)$ is continuous, it follows that a unique minimum (resp. maximum) is attained at the upper (resp. lower) bound of this interval. Second, if $\frac{\pi}{2} \leq \theta_{\infty}<\pi$, then the mapping remains bijective, but from $\left.] 0, \frac{\pi}{2}-\frac{\theta_{\infty}}{2}\right]$ to $[1,+\infty[$, since

$$
\lim _{\theta_{0} \rightarrow 0^{+}} \frac{\sin \theta_{0}+\sin \theta_{\infty}+\sin \left(\theta_{0}+\theta_{\infty}\right)}{2 \sin \theta_{0} \sin \theta_{\infty} \sin \left(\theta_{0}+\theta_{\infty}\right)}=+\infty
$$

Hence, $\rho$ no longer has an upper bound. These results can be summarized as follows.

\section{Proposition 3.3.}

$$
\begin{aligned}
& \left(\forall \theta _ { \infty } \in \left[\frac{\pi}{3}, \pi[) \quad \inf _{\theta_{0}} \rho=\frac{1+\sin \frac{\theta_{\infty}}{2}}{\sin \theta_{\infty} \cos \frac{\theta_{\infty}}{2}},\right.\right. \\
& \left(\forall \theta _ { \infty } \in \left[\frac{\pi}{3}, \frac{\pi}{2}[) \quad \sup _{\theta_{0}} \rho=\frac{1+\cos \theta_{\infty}}{\sin \theta_{\infty} \sin 2 \theta_{\infty}} .\right.\right.
\end{aligned}
$$

Propositions 3.1 and 3.3 are illustrated in Figures 3 and 4
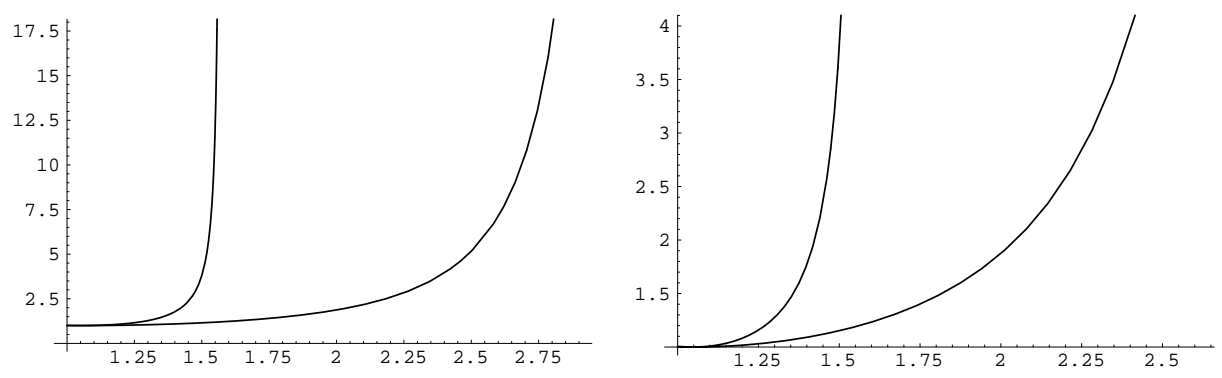

Figure 5. Graphs at two different scales providing the range of $\frac{\rho}{2}$ as a function of the maximal angle in radians; for $\theta_{\infty}>\frac{\pi}{2}$, the upper bound is not defined. 
Example 3.4. Let the maximal angle be set to $120^{\circ}$, which is a commonly accepted value, then the normalized radius-ratio can be as low as from circa 2.15 which is generally considered to be acceptable, but has no upper bound.

\section{EDGe RATio}

Another rather intuitive approach is to compare the extremal edges of the triangle by means of what we call the edge ratio, defined as

$$
\tau=\frac{|t|_{\infty}}{|t|_{0}}
$$

or, according to Lemma 2.1

$$
\tau=\frac{\sin \theta_{\infty}}{\sin \theta_{0}} .
$$

4.1. Extremum. It is clear that $\tau$ is a nondimensional measure, as $\rho$ is. Moreover, it is straightforward to see that $\tau \geq 1$, whereas the equality occurs if and only if the triangle is equilateral. Hence, $\tau$ shares the essential property of $\rho$, that its minimal value is attained for, and only for, an equilateral triangle.

4.2. Asymptotic behavior. The first-order derivatives of $\tau$ as expressed in (4.2) are

$$
\begin{aligned}
\frac{\partial \tau}{\partial \theta_{0}}\left(\theta_{0}, \theta_{\infty}\right) & =\frac{\cos \theta_{0} \sin \theta_{\infty}}{-\sin ^{2} \theta_{0}}, \\
\frac{\partial \tau}{\partial \theta_{\infty}}\left(\theta_{0}, \theta_{\infty}\right) & =\frac{\cos \theta_{\infty}}{\sin \theta_{0}} .
\end{aligned}
$$

Since $0<\theta_{0} \leq \frac{\pi}{3}$, $\frac{\partial \tau}{\partial \theta_{0}}$ never vanishes and thus $\tau$ has no stationary value over $\Omega$. In particular, this implies that the tangent plane at the minimal value is not horizontal. Therefore, the discrimination of configurations surrounding the equilateral triangle is sharper than with, for example, the radius-ratio.

We now examine how the bounds of $\tau$ depend on the minimal angle. We note that $\frac{\partial \tau}{\partial \theta_{0}}$ is strictly negative over $\Omega$. Therefore, $\theta_{0} \mapsto \tau\left(\theta_{0}, \theta_{\infty}\right)$ is monotonically decreasing for any particular value of $\theta_{\infty}$. In addition,

$$
\left(\forall \theta_{\infty} \in\left[\frac{\pi}{3}, \frac{\pi}{2}\right]\right) \quad \pi-2 \theta_{\infty} \leq \theta_{0} \leq \frac{\pi-\theta_{\infty}}{2}
$$

and

$$
\left(\forall \theta _ { \infty } \in \left[\frac{\pi}{2}, \pi[) \quad 0 \leq \theta_{0} \leq \frac{\pi-\theta_{\infty}}{2},\right.\right.
$$

whence the following properties can be asserted:

\section{Proposition 4.1.}

$$
\begin{aligned}
& \left(\forall \theta _ { \infty } \in \left[\frac{\pi}{3}, \pi[) \quad \inf _{\theta_{0}} \tau=2 \sin \frac{\theta_{\infty}}{2},\right.\right. \\
& \left(\forall \theta _ { \infty } \in \left[\frac{\pi}{3}, \frac{\pi}{2}[) \quad \sup _{\theta_{0}} \tau=\frac{1}{2 \cos \theta_{\infty}} .\right.\right.
\end{aligned}
$$

In addition, when $\theta_{\infty} \geq \frac{\pi}{2}, \tau$ is upper unbounded. These properties are illustrated in Figure 6]

The maximal angle dependency requires a more detailed discussion. In fact, the sign of $\frac{\partial \tau}{\partial \theta_{\infty}}$ is given by that of $\cos \theta_{\infty}$ (i.e., it is positive if and only if the maximal angle is acute). Moreover, $\theta_{\infty}$ has to comply with (2.3), thus $\frac{\pi}{2}$ belongs to 

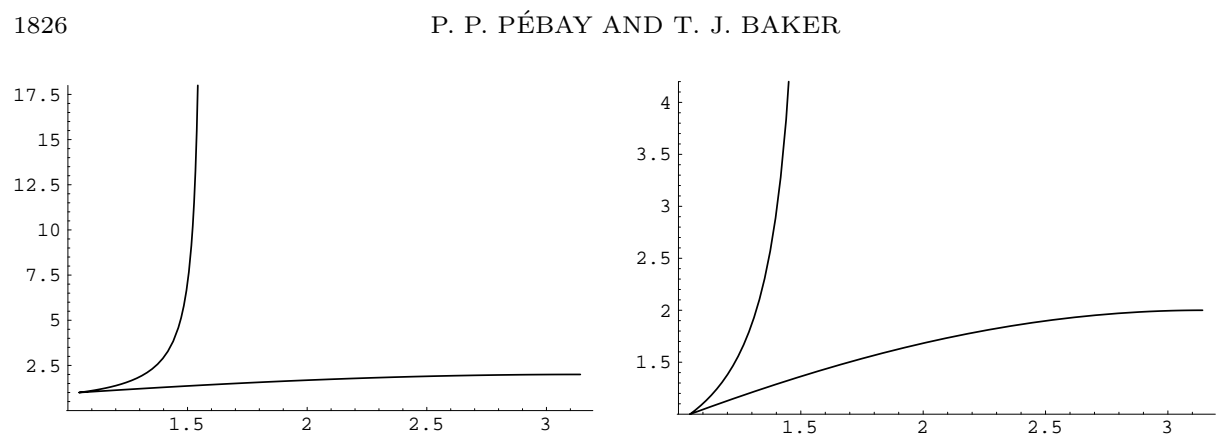

FiguRE 6. Graphs at two different scales providing the range of $\tau$ as a function of the maximal angle in radians; for $\theta_{\infty}>\frac{\pi}{2}$, the upper bound is not defined.

$\left[\frac{\pi}{2}-\frac{\theta_{0}}{2}, \pi-\theta_{0}\right]$ if and only if $\theta_{0} \leq \frac{\pi}{4}$. Therefore, $\theta_{\infty} \mapsto \tau\left(\theta_{0}, \theta_{\infty}\right)$ is monotonically increasing for any particular $\theta_{0} \geq \frac{\pi}{4}$. Taking into account the continuity of $\tau$, the extrema arise immediately. When $\theta_{0} \geq \frac{\pi}{4}$, the monotonicity is no longer true; more precisely, $\theta_{\infty} \mapsto \tau\left(\theta_{0}, \theta_{\infty}\right)$ reaches a maximum, $\frac{1}{\sin \theta_{0}}$ when $\theta_{\infty}=\frac{\pi}{2}$. One can observe that this value is indeed equal to $2 \cos \theta_{0}$ when the minimal angle is $\frac{\pi}{4}$, as expected. Concerning the minimum, the only difficulty comes from the fact that one, $\frac{1}{2 \sin \frac{\theta_{0}}{2}}$, is attained at $\frac{\pi}{2}-\frac{\theta_{0}}{2}$ and another one, $2 \cos \theta_{0}$, at $\pi-2 \theta_{0}$. Now, since $\sin \theta_{0}>0$

$$
\begin{aligned}
\tau\left(\theta_{0}, \frac{\pi}{2}-\frac{\theta_{0}}{2}\right) \leq \tau\left(\theta_{0}, \pi-2 \theta_{0}\right) & \Longleftrightarrow \cos \frac{\theta_{0}}{2} \leq \sin 2 \theta_{0} \\
& \Longleftrightarrow \cos \frac{\theta_{0}}{2} \leq \cos \left(2 \theta_{0}-\frac{\pi}{2}\right) \\
& \Longleftrightarrow \cos \left(2 \theta_{0}-\frac{\pi}{2}\right)-\cos \frac{\theta_{0}}{2} \geq 0 \\
& \Longleftrightarrow \sin \left(\frac{5 \theta_{0}-\pi}{4}\right) \sin \left(\frac{3 \theta_{0}-\pi}{4}\right) \leq 0
\end{aligned}
$$

In addition, $0<\frac{\theta_{0}}{3} \leq \frac{\pi}{3}$, whence

$$
\tau\left(\theta_{0}, \frac{\pi}{2}-\frac{\theta_{0}}{2}\right) \leq \tau\left(\theta_{0}, \pi-2 \theta_{0}\right) \quad \Longleftrightarrow \quad \sin \left(\frac{5 \theta_{0}-\pi}{4}\right) \leq 0 .
$$

Given the range of the minimal angle, (4.13) is satisfied if and only if $\theta_{0} \geq \frac{\pi}{5}$. We can hence conclude:

\section{Proposition 4.2.}

$$
\begin{array}{ll}
\left.\left.\left(\forall \theta_{0} \in\right] 0, \frac{\pi}{5}\right]\right) \quad \inf _{\theta_{\infty}} \tau=2 \cos \theta_{0}, \\
\left(\forall \theta_{0} \in\left[\frac{\pi}{5}, \frac{\pi}{3}\right]\right) \quad \inf _{\theta_{\infty}} \tau=\frac{1}{2 \sin \frac{\theta_{0}}{2}}, \\
\left(\forall \theta_{0} \in\left[0, \frac{\pi}{4}\right]\right) \quad \sup _{\theta_{\infty}} \tau=\frac{1}{\sin \theta_{0}}, \\
\left(\forall \theta_{0} \in\left[\frac{\pi}{4}, \frac{\pi}{3}\right]\right) \quad \sup _{\theta_{\infty}} \tau=2 \cos \theta_{0} .
\end{array}
$$

This beautiful distribution of extrema is displayed Figure 7 

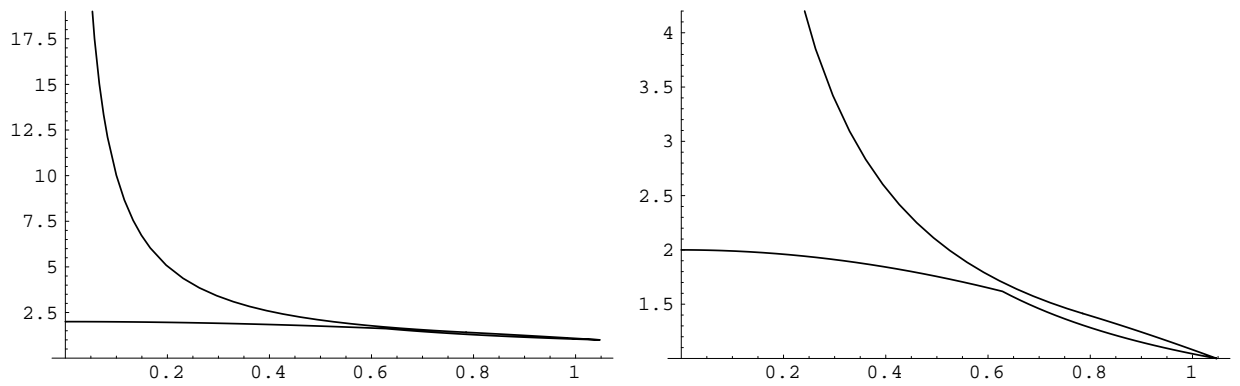

Figure 7. Graphs at two different scales providing the range of $\tau$ as a function of the minimal angle in radians.

\section{Edge to CIRCUMradius}

We consider the following two ratios:

$$
\omega=\frac{R}{|t|_{\infty}}, \quad \frac{R}{|t|_{0}} .
$$

Then (1.1) and Lemma 2.1 give

$$
\omega=\frac{1}{2 \sin \theta_{\infty}}, \quad \frac{R}{|t|_{0}}=\frac{1}{2 \sin \theta_{0}} .
$$

The main problem with the two latter ratios lies in the fact that they take into account only one edge, which has the consequence that the nice symmetry properties of $\rho$ and $\tau$ are lost. In order to avoid this problem while keeping a nondimensional quantity, it is natural to use the perimeter or, since this quantity better fits with classic triangle metric formulas, the half-perimeter. In fact, (1.1) gives

$$
\nu=\frac{R}{p}=\frac{2 R}{a+b+c}=\frac{1}{\sin \alpha+\sin \beta+\sin \gamma},
$$

which, in terms of the extremal angles, can be written as

$$
\nu=\frac{1}{\sin \theta_{0}+\sin \theta_{\infty}+\sin \left(\theta_{0}+\theta_{\infty}\right)} .
$$

5.1. Extremum. The mapping $x \mapsto \frac{1}{\sin x}$ strictly decreases (resp. increases) over ] $\left.0, \frac{\pi}{2}\right]$ (resp. $\left[\frac{\pi}{2}, \pi\left[\right.\right.$ ); therefore, since $\theta_{0}\left(\right.$ resp. $\left.\theta_{\infty}\right)$ ranges over $\left.] 0, \frac{\pi}{3}\right]$ (resp. $\left[\frac{\pi}{3}, \pi[)\right.$, $\frac{R}{|t|_{0}}$ (resp. $\left.\omega\right)$ attains its unique minimum at equilateral (resp. right) triangles. In addition, $\frac{\pi}{2}$ is a stationary value.

From these considerations, it becomes clear that, except for particular applications which require right angles, $\omega$ is not a quality measurement in the usual sense of assigning a high grade to equilateral triangles. Moreover, each of them is only related to one extremal angle; this is problematic especially for $\omega$, which confuses needles with "good" right triangles.

Now, since $0<\theta_{0} \leq \frac{\pi}{3}$, (5.2) makes it clear that $\theta_{0} \mapsto \frac{R}{\left.t t\right|_{0}}$ is bijective; therefore, the properties of this ratio can be directly deduced from those of the minimal angle through

$$
\theta_{0}=\arcsin \frac{|t|_{0}}{2 R}
$$


In particular, $\frac{R}{|t|_{0}}$ and $\rho$ both attain their absolute minimum for an equilateral triangle.

Now, since the denominator in (5.4) is strictly positive, $\nu$ is minimal if and only if

$$
\sin \theta_{0}+\sin \theta_{\infty}+\sin \left(\theta_{0}+\theta_{\infty}\right)=g\left(\theta_{0}, \theta_{\infty}\right)
$$

attains a maximum. For the same reasons as for $\rho$, the necessary condition for an extremum is

$$
\left\{\begin{aligned}
\cos \theta_{0}+\cos \left(\theta_{0}+\theta_{\infty}\right) & =0 \\
\cos \theta_{\infty}+\cos \left(\theta_{0}+\theta_{\infty}\right) & =0
\end{aligned}\right.
$$

which implies $\cos \theta_{0}=\cos \theta_{\infty}$, thus $\theta_{0}=\theta_{\infty}$, since these two angles are in $] 0, \pi[$. Moreover, they have to comply with both (2.1) and (2.3), hence $\theta_{0}=\theta_{\infty}=\frac{\pi}{3}$. In addition, the hessian determinant at any point is

$$
\sin \theta_{\infty} \sin \theta_{0}+\left(\sin \theta_{0}+\sin \theta_{\infty}\right) \sin \left(\theta_{0}+\theta_{\infty}\right)
$$

which is strictly positive, since $\theta_{0}, \theta_{\infty}$ and $\theta_{0}+\theta_{\infty}$ are in $] 0, \pi[$. Lastly,

$$
\frac{\partial^{2} \nu}{\partial \theta_{0}^{2}}\left(\theta_{0}, \theta_{\infty}\right)=-\sin \theta_{0}-\sin \left(\theta_{0}+\theta_{\infty}\right)<0
$$
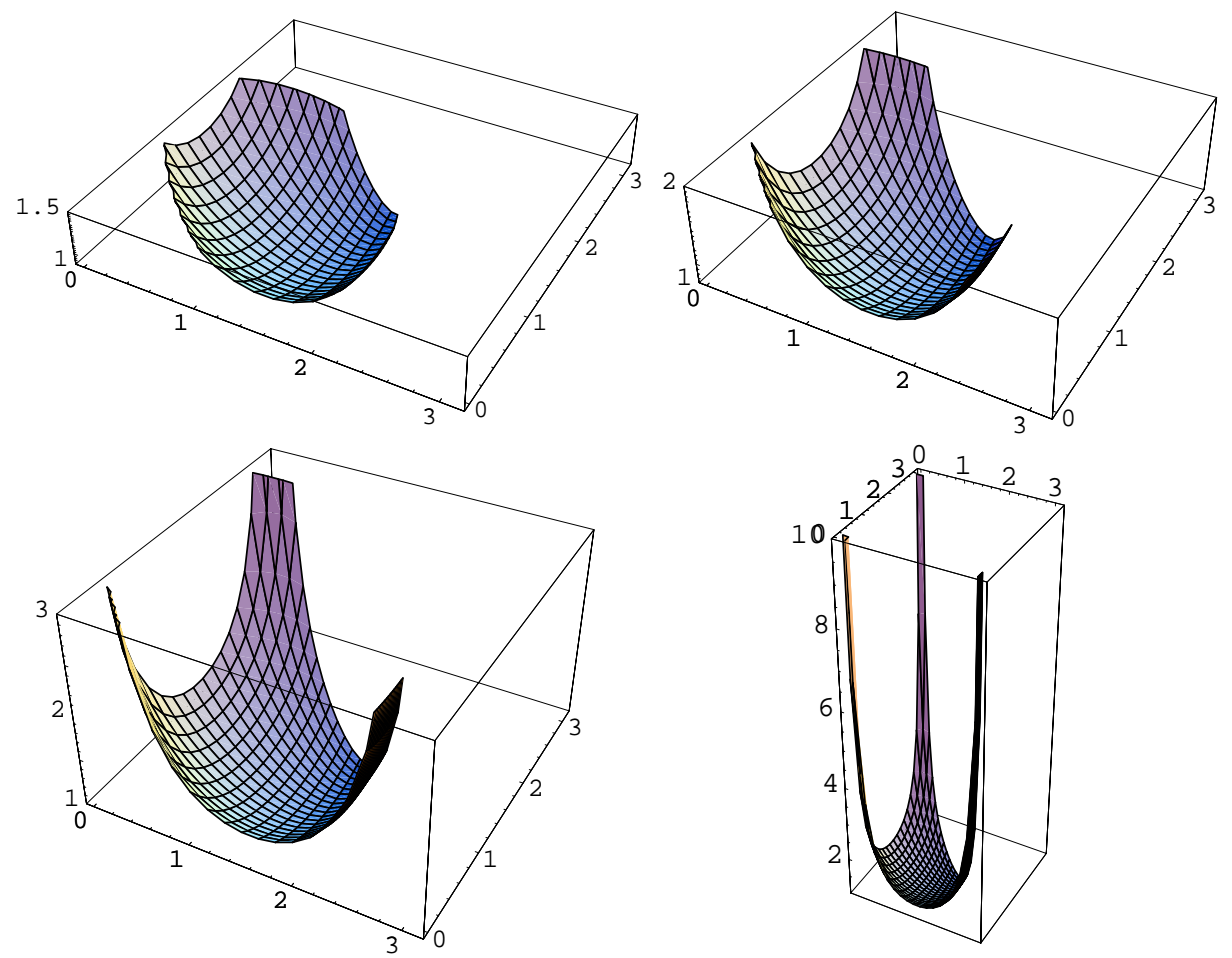

Figure 8. Surface $z=\frac{3 \sqrt{3}}{2} \nu$ as a function of two angles in radians: (i) $z<1.5$; (ii) $z<2$; (iii) $z<3$; (iv) $z<10$. 
which means that $\nu$ is concave over the domain. Hence, its unique stationary point is also its unique absolute maximum; in other words, $\nu$ is minimal if and only if the triangle is equilateral, as illustrated by Figure 8 .

5.2. Asymptotic behavior. In addition, Figure 8 also clearly suggests that, whereas $\rho$ and $\nu$ have a similar asymptotic behavior for flattened triangles, this is not the case for needles. More precisely, it follows from (5.7) that

$$
\frac{\partial \nu}{\partial \theta_{0}}\left(\theta_{0}, \theta_{\infty}\right) \geq 0 \Longleftrightarrow \theta_{0}+\theta_{\infty} \leq \pi-\theta_{0},
$$

which is satisfied because of (2.1). Whence, using the same arguments as for $\rho$,

Proposition 5.1.

$$
\begin{aligned}
& \left(\forall \theta _ { \infty } \in \left[\frac{\pi}{3}, \pi[) \quad \inf _{\theta_{0}} \nu=\frac{1}{2 \cos \frac{\theta_{\infty}}{2}+\sin \theta_{\infty}},\right.\right. \\
& \left(\forall \theta_{\infty} \in\left[\frac{\pi}{3}, \frac{\pi}{2}\right]\right) \quad \sup _{\theta_{0}} \nu=\frac{1}{2 \sin \theta_{\infty}+\sin 2 \theta_{\infty}}, \\
& \left(\forall \theta _ { \infty } \in \left[\frac{\pi}{2}, \pi[) \quad \sup _{\theta_{0}} \nu=\frac{1}{2 \sin \theta_{\infty}}=\omega .\right.\right.
\end{aligned}
$$

We also observe that (5.7) implies that

$$
\frac{\partial \nu}{\partial \theta_{\infty}}\left(\theta_{0}, \theta_{\infty}\right) \geq 0 \Longleftrightarrow \theta_{0}+\theta_{\infty} \leq \pi-\theta_{\infty},
$$

which is never true because of (2.3) $)$. Thus,

\section{Proposition 5.2.}

$$
\begin{aligned}
& \left.\left.\left(\forall \theta_{0} \in\right] 0, \frac{\pi}{3}\right]\right) \quad \inf _{\theta_{\infty}} \nu=\frac{1}{2 \cos \frac{\theta_{0}}{2}+\sin \theta_{0}}, \\
& \left.\left.\left(\forall \theta_{0} \in\right] 0, \frac{\pi}{3}\right]\right) \quad \sup _{\theta_{\infty}} \nu=\frac{1}{2 \sin \theta_{0}+\sin 2 \theta_{0}} .
\end{aligned}
$$

As illustrated by Figure 9, the range of $\nu$ depending on $\theta_{\infty}$ is extremely narrow compared to $\rho$, which confirms the intuitive idea of a measurement driven by the maximal angle, but which remains symmetric. In fact, the important point is the supremum: it is equal to $\frac{1}{2}$ (to $\frac{3 \sqrt{3}}{4}$ for the normalized $\nu$, as displayed in the figures) when $\theta_{\infty}=\frac{\pi}{2}$, hence needles are considered to be good elements by $\nu$, which makes
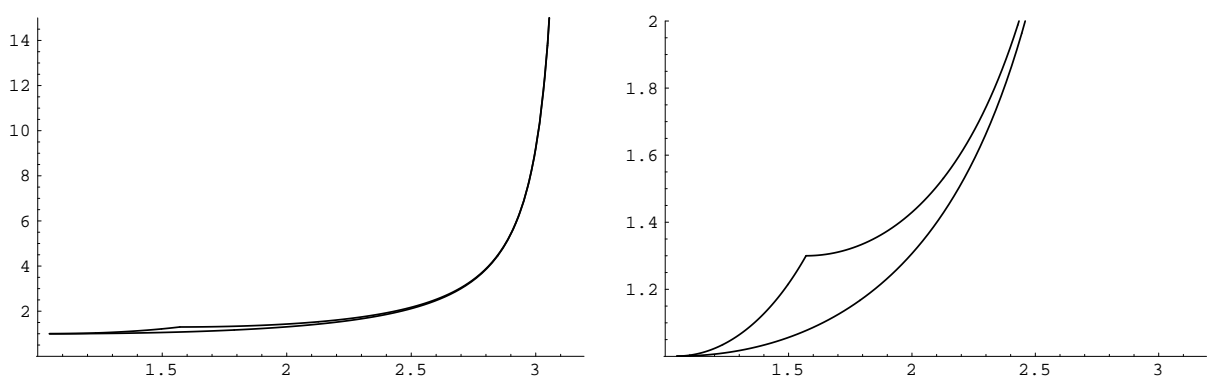

Figure 9. Graphs at two different scales providing the range of $\frac{3 \sqrt{3}}{2} \nu$ as a function of the maximal angle in radians. 

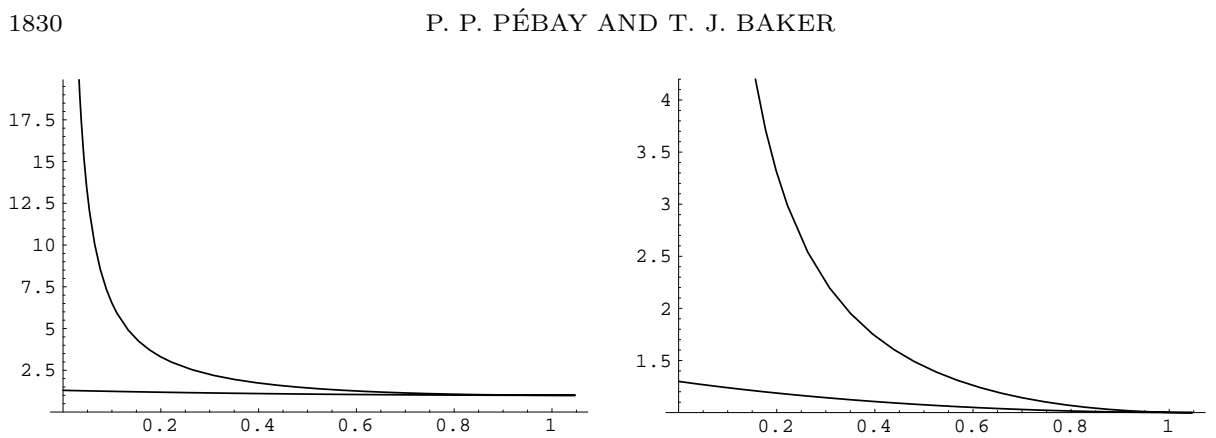

FiguRE 10. Graphs at two different scales providing the range of $\frac{3 \sqrt{3}}{2} \nu$ as a function of the minimal angle in radians.
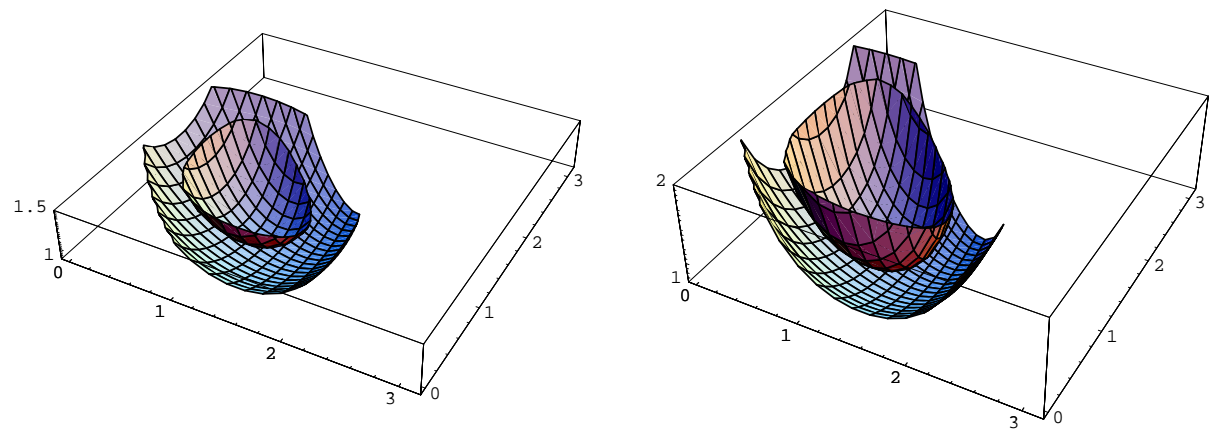

Figure 11. Surfaces $z=\frac{\rho}{2}$ (interior) and $z=\frac{3 \sqrt{3}}{2} \nu$ (exterior) as functions of two angles in radians: (i) $z<1.5$; (ii) $z<2$.

another difference with $\rho$. Moreover, and this is the essential difference with $\frac{R}{\left|t_{\infty}\right|}$, while these needles are considered acceptable, they are not assumed to be the best possible configuration. These remarks are confirmed when examining Figure $10 \nu$ does not provide any discriminant information for small minimal angles, since they can occur both in needles and flattened triangles. For these reasons, we tend to consider $\nu$ to be an excellent measurement.

Finally, it is also interesting to compare the variations of $\rho$ and $\nu$ in the neighborhood of equilateral triangles. This is illustrated by Figure 11, which shows both normalized measurements for values not bigger than 2 (i.e., for good quality elements in the sense of the corresponding measurement). It is clear that $\rho$ distinguishes angles much better than $\nu$ does. Hence, these two measurements complement each other in the following way: first, $\rho$ discriminates good triangles (i.e., at a user-defined distance from equilateral); second, among the remaining ones, $\nu$ is able to sort the needles.

\section{EdGe TO INRADIUS}

Finally, we compare edge lengths with the inradius:

$$
\zeta=\frac{p}{r} .
$$


It follows from (3.5) and (5.3) that

$$
\begin{aligned}
\zeta & =\frac{\rho}{\nu}=\frac{(\sin \alpha+\sin \beta+\sin \gamma)^{2}}{2 \sin \alpha \sin \beta \sin \gamma} \\
& =\frac{\left(\sin \theta_{0}+\sin \theta_{\infty}+\sin \left(\theta_{0}+\theta_{\infty}\right)\right)^{2}}{2 \sin \theta_{0} \sin \theta_{\infty} \sin \left(\theta_{0}+\theta_{\infty}\right)}
\end{aligned}
$$

Because it is often considered to be the standard quality measurement for finite element error estimates, we also say a word about

$$
\iota=\frac{|t|_{\infty}}{r},
$$

often referred to as the aspect ratio 2 First, as for $\omega$, the symmetry properties vanish; this affects computational efficiency, since it implies that two tests must be performed in order to decide which is the largest angle or the longest edge. Second, (3.19) and (5.2) lead to

$$
\iota=\frac{\sin \theta_{0}+\sin \theta_{\infty}+\sin \left(\theta_{0}+\theta_{\infty}\right)}{\sin \theta_{0} \sin \left(\theta_{0}+\theta_{\infty}\right)},
$$

which can be rewritten in a more convenient way just by replacing $\sin \theta_{\infty}$ by $\sin \left(\theta_{0}+\theta_{\infty}-\theta_{0}\right)$, as

$$
\iota\left(\theta_{0}, \theta_{\infty}\right)=\tan \frac{\theta_{0}+\theta_{\infty}}{2}+\cot \frac{\theta_{0}}{2} .
$$

6.1. Extremum. It is not necessarily useful to begin a full study of $\zeta$, since what we already know about $\rho$ and $\nu$ allows us to directly summarize its main properties in Table 1

For good quality triangles, $\rho, \nu$ and $\zeta$ give equivalent results. More precisely, combining (1.3) and (1.2) gives

$$
\zeta=\sqrt{\frac{p^{3}}{(p-a)(p-b)(p-c)}},
$$

whence $\zeta$ is minimal when $\frac{(p-a)(p-b)(p-c)}{p^{3}}$ is maximal. This expression is, of course, a function of only the three variables $a, b$ and $c$, which can be expressed as

$$
h(a, b, c)=\frac{(b+c-a)(a+b-c)(a+c-b)}{(a+b+c)^{3}},
$$

TABLE 1. Qualitative values of various measurements for the three triangle categories.

\begin{tabular}{|c||c|c|c|}
\hline & good & needle & flattened \\
\hline$\frac{1}{2} \rho$ & $\sim 1$ & $\gg 1$ & $\gg 1$ \\
\hline$\frac{3 \sqrt{3}}{2} \nu$ & $\sim 1$ & $\sim \frac{3 \sqrt{3}}{4}$ & $\gg 1$ \\
\hline$\frac{1}{3 \sqrt{3}} \zeta$ & $\sim 1$ & $\gg 1$ & $?$ \\
\hline
\end{tabular}

\footnotetext{
${ }^{2}$ However, since there is no uniform nomenclature in this field, we do not use this term in order to avoid confusion and instead simply refer to it as $\iota$.
} 
and it is clear that $h\left(1, \frac{b}{a}, \frac{c}{a}\right)=h(a, b, c)$ for any $a \in \mathbb{R}_{+}^{*}$. This means that $h$ only depends on two variables, given by the ratios of two edge lengths to the third one. In other words, $h$ is invariant through homotopy, as expected, since $\zeta$ is nondimensional. We therefore study the variations of

$$
\begin{array}{rllc}
\tilde{h}: & \mathbb{R}_{+}^{* 2} & \longrightarrow & \mathbb{R}, \\
(x, y) & \longmapsto & \frac{(x+y-1)(x+1-y)(y+1-x)}{(x+y+1)^{3}} .
\end{array}
$$

The first-order derivatives are given by

$$
\begin{aligned}
& \frac{\partial \tilde{h}}{\partial x}(x, y)=\frac{4\left(1-y-y^{2}+y^{3}+2 y x-(1+y) x^{2}\right)}{(1+x+y)^{4}} \\
& \frac{\partial \tilde{h}}{\partial y}(x, y)=\frac{4\left(1-x-x^{2}+x^{3}+2 y x-(1+x) y^{2}\right)}{(1+x+y)^{4}}
\end{aligned}
$$

whence the stationary point condition implies that $(x-y)\left((x+y)^{2}-1\right)=0$ (i.e., $x=y$ or $x=1-y$ ). If $x=y$, then $x=y=1$, while assuming $x=1-y$ in $\frac{\partial \tilde{h}}{\partial x}=0$ implies that $y=0$, which is impossible. Furthermore, the calculation of the hessian determinant at $(1,1)$ returns a positive number, while $\frac{\partial^{2} \tilde{h}}{\partial x^{2}}<0$. Hence, for the same reasons as previously, since $\mathbb{R}_{+}^{* 2}$ is a domain, $\zeta$ reaches its only extremum, which is a minimum, for equilateral triangles.

For the ratio $\iota$, the first-order derivatives are given by

$$
\begin{aligned}
\frac{\partial \iota}{\partial \theta_{0}}\left(\theta_{0}, \theta_{\infty}\right) & =\frac{-\cos \frac{\theta_{\infty}}{2} \cos \frac{2 \theta_{0}+\theta_{\infty}}{2}}{2 \sin ^{2} \frac{\theta_{0}}{2} \cos ^{2} \frac{\theta_{0}+\theta_{\infty}}{2}}, \\
\frac{\partial \iota}{\partial \theta_{\infty}}\left(\theta_{0}, \theta_{\infty}\right) & =\frac{1}{2 \cos ^{2} \frac{\theta_{0}+\theta_{\infty}}{2}} .
\end{aligned}
$$

Hence $\iota$ has no stationary point, thus no extremum over the interior of $\Omega$, while some may exist on the boundary $\partial \Omega$. Now (6.6) can be written on the upper edge of $\partial \Omega$ as $\theta_{0} \mapsto 2 \cot \frac{\theta_{0}}{2}$, which is clearly minimal when $\theta_{0}=\frac{\pi}{3}=\theta_{\infty}$. On the lower edge, the situation is a bit trickier, since $\iota$ becomes $\theta_{\infty} \mapsto \tan \theta_{\infty}+\cot \frac{\theta_{\infty}}{2}$, hence its derivative is

$$
\theta_{\infty} \longmapsto \frac{1}{\cos ^{2} \theta_{\infty}}-\frac{1}{2 \sin ^{2} \frac{\theta_{\infty}}{2}},
$$

the sign of which is given by that of $2 \sin ^{2} \frac{\theta_{\infty}}{2}-\cos ^{2} \theta_{\infty}$, which can be rewritten as $1-\cos \theta_{\infty}-\cos ^{2} \theta_{\infty}$. Now $\left.\left.\cos \theta_{\infty} \in\right]-1, \frac{1}{2}\right]$, since $\theta_{\infty} \in\left[\frac{\pi}{3}, \pi[\right.$. In addition, the polynomial $-X^{2}-X+1$ obviously has two distinct real roots, and is positive between $\frac{-\sqrt{5}-1}{2}<-1$ and $\frac{\sqrt{5}-1}{2}>\frac{1}{2}$. Hence, the derivative is strictly positive for any $\theta_{\infty}$, and this proves that the minimum of $\iota$ on the lower boundary is obtained for $\theta_{\infty}=\frac{\pi}{3}=\theta_{0}$.

6.2. Asymptotic behavior. Concerning $\zeta$, the last point to be addressed is the behavior for flattened triangles. Without loss of generality, one can make $a \approx 2 b \approx$ $2 c$ in (6.7), which gives that $\zeta \gg 1$. Hence, $\rho$ and $\zeta$ have basically the same behavior and the first one, being easier to compute, should be preferred.

The asymptotic behavior of $\iota$ can be found from (6.12) (resp. (6.13)), which shows that $\theta_{0} \mapsto \iota\left(\theta_{0}, \theta_{\infty}\right)$ (resp. $\left.\theta_{\infty} \mapsto \iota\left(\theta_{0}, \theta_{\infty}\right)\right)$ is always strictly monotonically 
decreasing (resp. increasing). Since it is also continuous, we have the following results:

\section{Proposition 6.1.}

$$
\begin{array}{ll}
\left.\left.\left(\forall \theta_{0} \in\right] 0, \frac{\pi}{3}\right]\right) & \inf _{\theta_{\infty}} \iota=\cot \frac{\theta_{0}}{2}+\tan \frac{\theta_{0}+\pi}{4} \\
\left.\left.\left(\forall \theta_{0} \in\right] 0, \frac{\pi}{3}\right]\right) & \sup _{\theta_{\infty}} \iota=2 \cot \frac{\theta_{0}}{2}
\end{array}
$$

and

\section{Proposition 6.2.}

$$
\begin{aligned}
& \left(\forall \theta _ { \infty } \in \left[\frac{\pi}{3}, \pi[) \quad \inf _{\theta_{0}} \iota=2 \frac{1+\sin \frac{\theta_{\infty}}{2}}{\cos \frac{\theta_{\infty}}{2}},\right.\right. \\
& \left(\forall \theta _ { \infty } \in \left[\frac{\pi}{3}, \frac{\pi}{2}[) \quad \sup _{\theta_{0}} \iota=2 \frac{1+\cos \theta_{\infty}}{\sin 2 \theta_{\infty}},\right.\right.
\end{aligned}
$$

while $\iota$ is not bounded above for $\theta_{\infty} \in\left[\frac{\pi}{2}, \pi[\right.$. The corresponding curves are provided by Figures 19 and 13.
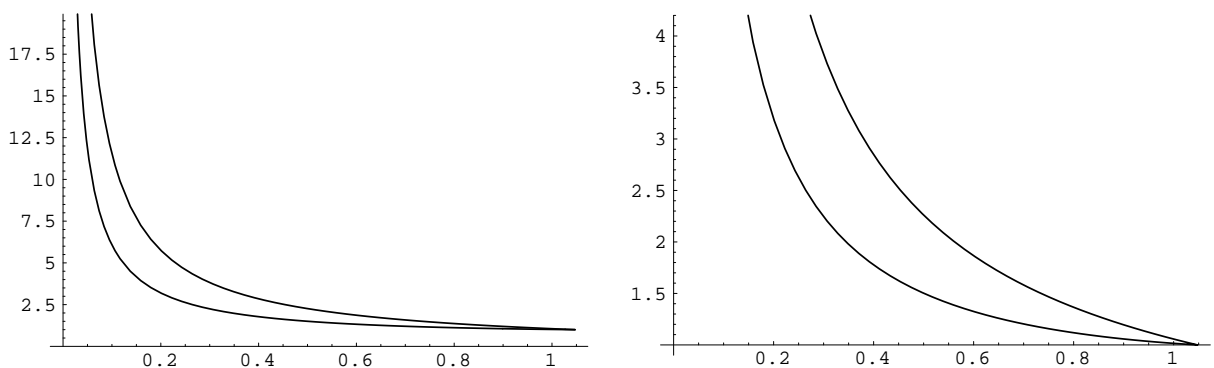

Figure 12. Graphs at two different scales providing the range of $\frac{\iota}{2 \sqrt{3}}$ as a function of the minimal angle in radians.
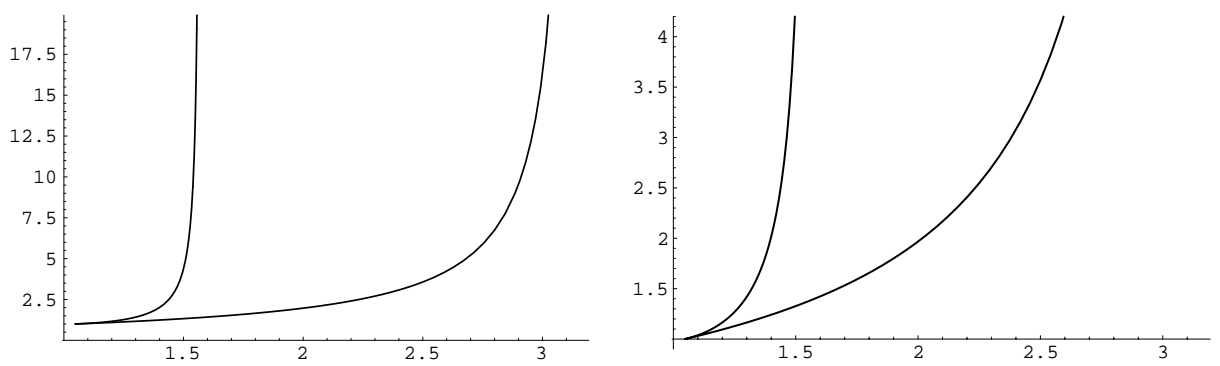

FiguRE 13. Graphs at two different scales providing the range of $\frac{\iota}{2 \sqrt{3}}$ as a function of the maximal angle in radians. 


\section{MATRIX NORMS}

As described in [3, 7, 9], we define an edge matrix of $t$ by

$$
T_{0}=\left(\begin{array}{cc}
x_{1}-x_{0} & x_{2}-x_{0} \\
y_{1}-y_{0} & y_{2}-y_{0}
\end{array}\right)
$$

and let $W$ be the edge matrix of a reference equilateral triangle, for example,

$$
W=\left(\begin{array}{cc}
1 & \frac{1}{2} \\
0 & \frac{\sqrt{3}}{2}
\end{array}\right) .
$$

We now let $A_{0}=T_{0} W^{-1}$ be the matrix that maps $W$ into $T_{0}$ and define matrix norms based on the singular values $\sigma$ of $A_{0}$. The singular values are given by the positive square roots of the eigenvalues of the positive definite matrix $A_{0}^{T} A_{0}$. Now

$$
A_{0}^{T} A_{0}=\left(\begin{array}{cc}
u & w \\
w & v
\end{array}\right),
$$

where

$$
\begin{gathered}
u=\left|\mathbf{v}_{1}-\mathbf{v}_{0}\right|^{2} \\
3 v=4\left|\mathbf{v}_{2}-\mathbf{v}_{0}\right|^{2}-4\left(\mathbf{v}_{2}-\mathbf{v}_{0}\right) \cdot\left(\mathbf{v}_{1}-\mathbf{v}_{0}\right)+\left|\mathbf{v}_{1}-\mathbf{v}_{0}\right|^{2}
\end{gathered}
$$

and

$$
\sqrt{3} w=2\left(\mathbf{v}_{2}-\mathbf{v}_{0}\right) \cdot\left(\mathbf{v}_{1}-\mathbf{v}_{0}\right)-\left|\mathbf{v}_{1}-\mathbf{v}_{0}\right|^{2} .
$$

In the above expressions, $\cdot$ denotes the usual scalar product. The singular values $\sigma$ of $A_{0}$ are thus obtained from the characteristic equation of $A_{0}^{T} A_{0}$ as

$$
\begin{aligned}
\frac{3}{4} \sigma^{4} & -\left(\left|\mathbf{v}_{1}-\mathbf{v}_{0}\right|^{2}+\left|\mathbf{v}_{2}-\mathbf{v}_{0}\right|^{2}-\left(\mathbf{v}_{2}-\mathbf{x}_{0}\right) \cdot\left(\mathbf{v}_{1}-\mathbf{v}_{0}\right)\right) \sigma^{2} \\
& +\left|\mathbf{v}_{1}-\mathbf{v}_{0}\right|^{2}\left|\mathbf{v}_{2}-\mathbf{x}_{0}\right|^{2}-\left(\left(\mathbf{v}_{2}-\mathbf{x}_{0}\right) \cdot\left(\mathbf{v}_{1}-\mathbf{v}_{0}\right)\right)^{2}=0 .
\end{aligned}
$$

Alternatively, this equation can be written as

$$
3 \sigma^{4}-2\left(a^{2}+b^{2}+c^{2}\right) \sigma^{2}+16 \mathcal{A}^{2}=0 .
$$

Hence,

$$
\sigma_{1}^{2}+\sigma_{2}^{2}=\frac{2}{3}\left(a^{2}+b^{2}+c^{2}\right)=\frac{2}{3}|t|_{2}^{2}
$$

and $\sigma_{1} \sigma_{2}=4 \frac{\mathcal{A}}{\sqrt{3}}$, where $\sigma_{1}^{2}$ and $\sigma_{2}^{2}\left(0<\sigma_{1} \leq \sigma_{2}\right)$ are the two roots of (7.8). A quality measure can be constructed from the condition number of any unitarily invariant norm of the matrix $A_{0}$ (cf. [3]). One such family is derived from the Schatten $p$-norms defined by

$$
N_{p}\left(A_{0}\right)=\left(\sigma_{1}^{p}+\sigma_{2}^{p}\right)^{1 / p}, \quad p \in[1,+\infty[.
$$

The case $p=2$ is the Frobenius norm, the limiting case $p \rightarrow \infty$ is the spectral norm, and the case $p=1$ is the trace norm. A nonnormalized quality measure is given by the condition number $\kappa_{p}\left(A_{0}\right)$, which is defined as

$$
\kappa_{p}\left(A_{0}\right)=\left[\left(\sigma_{1}^{p}+\sigma_{2}^{p}\right)\left(\sigma_{1}^{-p}+\sigma_{2}^{-p}\right)\right]^{1 / p} .
$$


For the particular case $p=2$, we find that

$$
\kappa_{2}=\frac{\sigma_{1}^{2}+\sigma_{2}^{2}}{\sigma_{1} \sigma_{2}}=\frac{a^{2}+b^{2}+c^{2}}{2 \sqrt{3} \mathcal{A}}=\frac{|t|_{2}^{2}}{2 \sqrt{3} \mathcal{A}},
$$

and from (1.1) it then follows that

$$
\begin{aligned}
\kappa_{2} & =\frac{\sin ^{2} \alpha+\sin ^{2} \beta+\sin ^{2} \gamma}{\sqrt{3} \sin \alpha \sin \beta \sin \gamma} \\
& =\frac{\sin ^{2} \theta_{0}+\sin ^{2} \theta_{\infty}+\sin ^{2}\left(\theta_{0}+\theta_{\infty}\right)}{\sqrt{3} \sin \theta_{0} \sin \theta_{\infty} \sin \left(\theta_{0}+\theta_{\infty}\right)} .
\end{aligned}
$$

The Frobenius norm has been investigated in [7, 9. Interestingly, this quality measure, more specifically its inverse called shape regularity, has been independently suggested by [4] in the context of mesh adaptation based on a posteriori error estimates.

For $p=1$, we get

$$
\kappa_{1}=\frac{\left(\sigma_{1}+\sigma_{2}\right)^{2}}{\sigma_{1} \sigma_{2}}=2+\kappa_{2},
$$

and for $p \rightarrow \infty$ we obtain

$$
\begin{aligned}
\kappa_{\infty} & =\frac{\sigma_{2}}{\sigma_{1}}=\frac{\sigma_{2}^{2}}{\sigma_{1} \sigma_{2}}=\frac{|t|_{2}^{2}+\sqrt{|t|_{2}^{4}-48 \mathcal{A}^{2}}}{4 \sqrt{3} \mathcal{A}} \\
& =\frac{1}{2} \kappa_{2}+\frac{1}{2} \sqrt{\kappa_{2}^{2}-4} .
\end{aligned}
$$

7.1. Extremum. From (7.15) and (7.17), we see that $\kappa_{1}$ and $\kappa_{\infty}$ are both monotonically increasing $\mathcal{C}^{\infty}(] 2,+\infty[)$ functions of $\kappa_{2}$ and can therefore be regarded as $\mathcal{C}^{\infty}$-equivalent to $\kappa_{2}$ and thus share the same extremal and asymptotic properties. Using (1.4), we see that

$$
\frac{\sigma_{1}^{p}+\sigma_{2}^{p}}{2} \geq\left(\sigma_{1}^{p} \sigma_{2}^{p}\right)^{1 / 2}
$$

and

$$
\frac{\sigma_{1}^{-p}+\sigma_{2}^{-p}}{2} \geq\left(\sigma_{1}^{-p} \sigma_{2}^{-p}\right)^{1 / 2}
$$

which imply that $\kappa_{p} \geq 2^{2 / p}$. Moreover, it is shown in [3] that this minimum is the only stationary value of $\kappa_{p}$, which is attained when $\sigma_{1}=\sigma_{2}$. When $p=1$ (resp. $p=2, p \rightarrow \infty$ ), it is obvious that the minimal value is 4 (resp. 2,1 ). The variation of $\kappa_{2}$ as a function of the two angles $\alpha$ and $\beta$ is plotted in Figure 14 .

7.2. Asymptotic behavior. It follows from (7.13) that

$$
\begin{aligned}
\frac{\partial \kappa_{2}}{\partial \theta_{0}}\left(\theta_{0}, \theta_{\infty}\right) & =\frac{-2 \sin \theta_{\infty} \sin \left(2 \theta_{0}+\theta_{\infty}\right)}{\sqrt{3} \sin ^{2} \theta_{0} \sin ^{2}\left(\theta_{0}+\theta_{\infty}\right)}, \\
\frac{\partial \kappa_{2}}{\partial \theta_{\infty}}\left(\theta_{0}, \theta_{\infty}\right) & =\frac{-2 \sin \theta_{0} \sin \left(\theta_{0}+2 \theta_{\infty}\right)}{\sqrt{3} \sin ^{2} \theta_{\infty} \sin ^{2}\left(\theta_{0}+\theta_{\infty}\right)} .
\end{aligned}
$$

In addition, (2.9) and (2.10) respectively imply that

$$
\left(\forall\left(\theta_{0}, \theta_{\infty}\right) \in \stackrel{\circ}{\Omega}\right) \quad \pi<\theta_{0}+2 \theta_{\infty}<2 \pi-3 \theta_{0}<2 \pi
$$



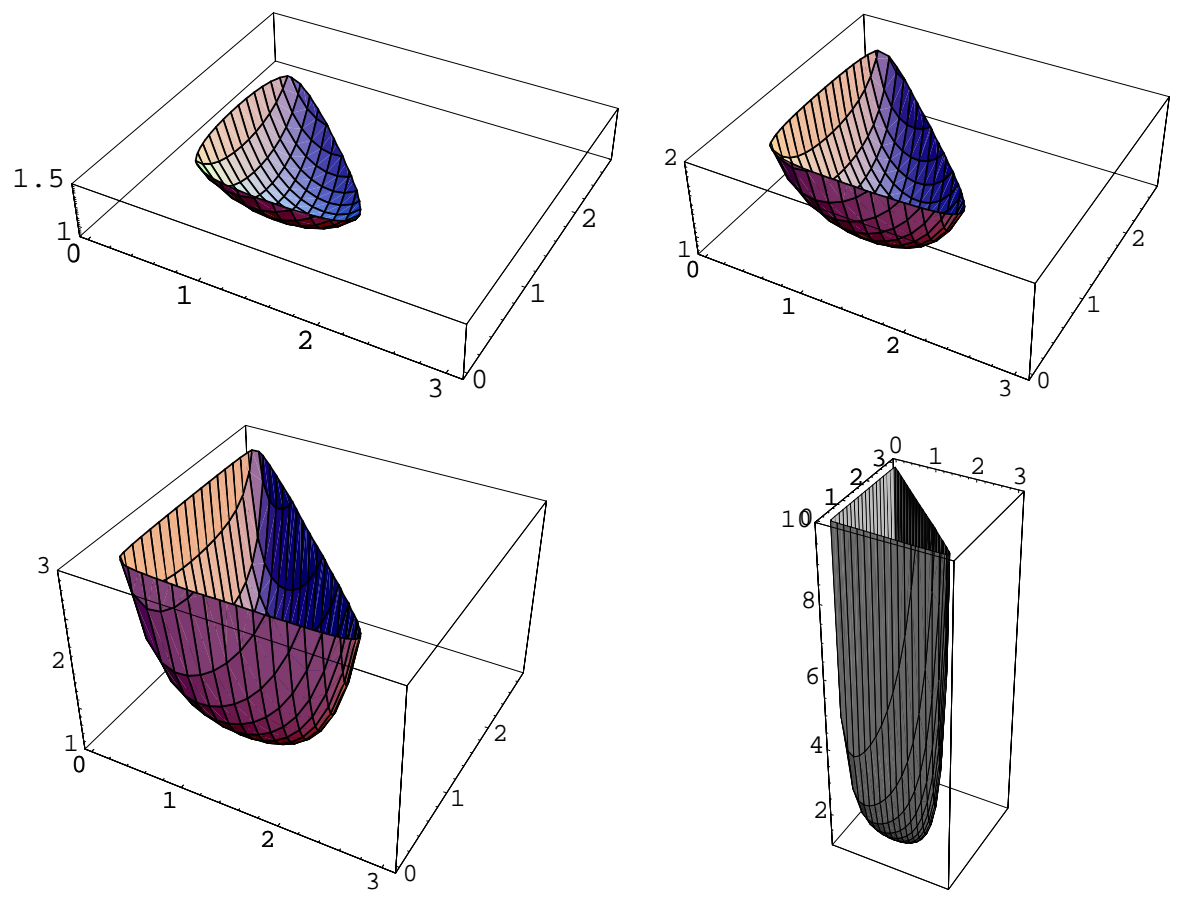

FIGURE 14. Surface $z=\frac{\kappa_{2}}{2}$ as a function of two angles in radians: (i) $z<1.5$; (ii) $z<2$; (iii) $z<3$; (iv) $z<10$.

and

$$
\left(\forall\left(\theta_{0}, \theta_{\infty}\right) \in \stackrel{\circ}{\Omega}\right) \quad 0<2 \theta_{0}+\theta_{\infty}<\pi .
$$

Therefore, for any $\theta_{\infty}$ in $\left[\frac{\pi}{3}, \pi\left[\right.\right.$ (resp. $\theta_{0}$ in $\left.] 0, \frac{\pi}{3}\right]$ ), the mapping $\theta_{0} \mapsto \kappa_{2}\left(\theta_{0}, \theta_{\infty}\right)$ (resp. $\theta_{\infty} \mapsto \kappa_{2}\left(\theta_{0}, \theta_{\infty}\right)$ ) is continuous and strictly monotonically decreasing (resp. increasing) over $\left.] \max \left(0, \pi-2 \theta_{\infty}\right), \frac{\pi}{2}-\frac{\theta_{\infty}}{2}\right]$ (resp. $\left[\frac{\pi}{2}-\frac{\theta_{0}}{2}, \pi-2 \theta_{0}\right]$ ), thus the following properties arise:

\section{Proposition 7.1.}

$$
\begin{array}{ll}
\left.\left.\left(\forall \theta_{0} \in\right] 0, \frac{\pi}{3}\right]\right) & \inf _{\theta_{\infty}} \kappa_{2}=\frac{2+4 \sin ^{2} \frac{\theta_{0}}{2}}{\sqrt{3} \sin \theta_{0}}, \\
\left.\left.\left(\forall \theta_{0} \in\right] 0, \frac{\pi}{3}\right]\right) & \sup _{\theta_{\infty} \kappa_{2}}=\frac{2+4 \cos ^{2} \theta_{0}}{\sqrt{3} \sin 2 \theta_{0}} .
\end{array}
$$

\section{Proposition 7.2.}

$$
\begin{aligned}
& \left(\forall \theta _ { \infty } \in \left[\frac{\pi}{3}, \pi[) \quad \inf _{\theta_{0}} \kappa_{2}=\frac{2+4 \sin ^{2} \frac{\theta_{\infty}}{2}}{\sqrt{3} \sin \theta_{\infty}},\right.\right. \\
& \left(\forall \theta _ { \infty } \in \left[\frac{\pi}{3}, \frac{\pi}{2}[) \quad \sup _{\theta_{0}} \kappa_{2}=\frac{2+4 \cos ^{2} \theta_{\infty}}{\sqrt{3} \sin 2 \theta_{\infty}} .\right.\right.
\end{aligned}
$$

In addition, when $\theta_{\infty} \geq \frac{\pi}{2}, \kappa_{2}$ is not bounded above. These results are illustrated by Figures 15 and 16 

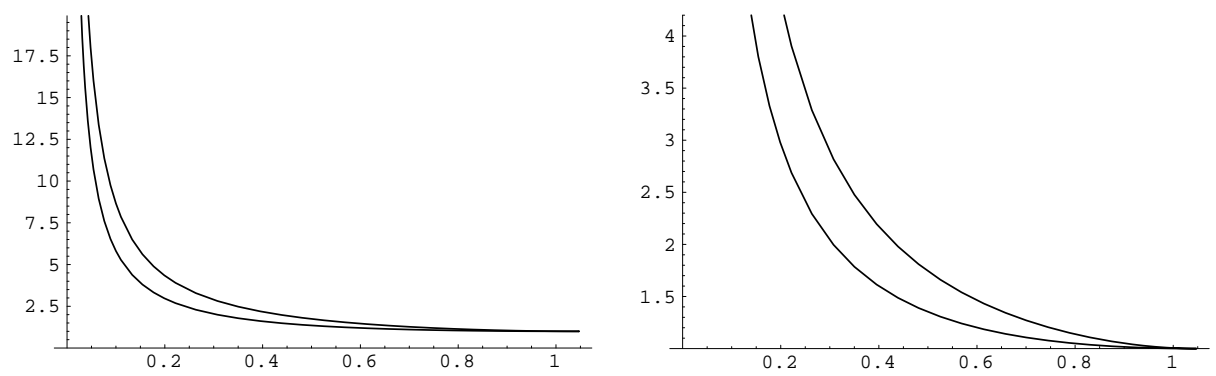

FiguRE 15. Graphs at two different scales providing the range of $\frac{\kappa_{2}}{2}$ as a function of the minimal angle in radians.
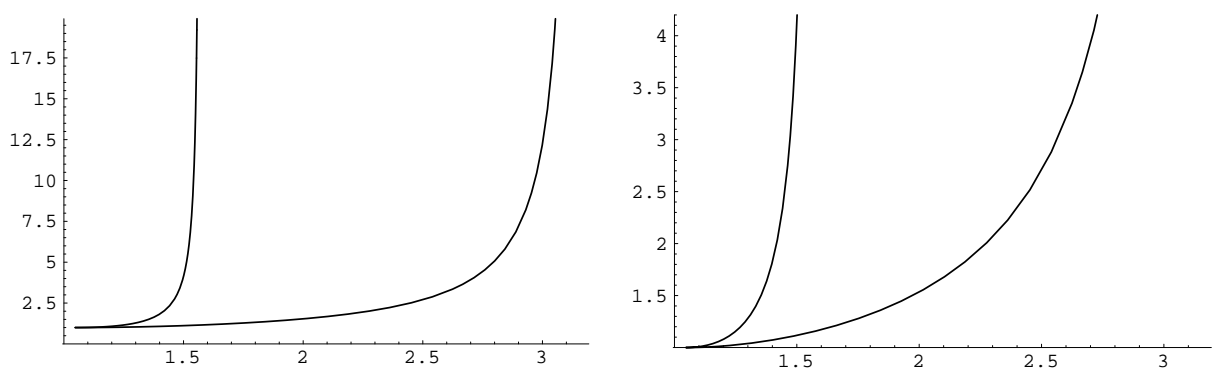

FiguRE 16. Graphs at two different scales providing the range of $\frac{\kappa_{2}}{2}$ as a function of the maximal angle in radians; for $\theta_{\infty}>\frac{\pi}{2}$, the upper bound is not defined.

\section{INEQUALITIES}

8.1. $\rho$ and $\tau$. According to (1.1), it is obvious that $2 R>|t|_{\infty}$. On the other hand, the assumption that $2 r \geq|t|_{0}$ implies that the two longest edges cannot meet at the third vertex, since they are tangent to the in-circle. This contradiction shows that $2 r<|t|_{0}$. Hence, for any nondegenerate triangle, one always has

$$
\tau<\rho .
$$

Let us assume that $x$ is the length of the side opposite the middle-angle $\theta$ of $t$. Then $x \leq|t|_{\infty}$ and $x \geq|t|_{0}$, hence

$$
\begin{aligned}
x^{2} & =|t|_{\infty}^{2}+|t|_{0}^{2}-2|t|_{\infty}|t|_{0} \cos \theta \\
& \Rightarrow\left\{\begin{array}{l}
x^{2}-|t|_{\infty}^{2}=|t|_{0}^{2}-2|t|_{\infty}|t|_{0} \cos \theta \leq 0 \\
x^{2}-|t|_{0}^{2}=|t|_{\infty}^{2}-2|t|_{\infty}|t|_{0} \cos \theta \geq 0
\end{array}\right. \\
& \Rightarrow \frac{1}{2 \tau} \leq \cos \theta \leq \frac{\tau}{2} .
\end{aligned}
$$

Hence, since $t \mapsto \arccos t$ is monotonically decreasing over $[0, \pi]$,

$$
\theta \leq \arccos \frac{1}{2 \tau}
$$

and, if $\tau \leq 2$,

$$
\theta \geq \arccos \frac{\tau}{2}
$$


In particular, it is easy to check that

$$
\theta=\arccos \frac{\tau}{2} \quad \Rightarrow \quad x=|t|_{0}
$$

and

$$
\theta=\arccos \frac{1}{2 \tau} \quad \Rightarrow \quad x=|t|_{\infty} .
$$

8.2. $\kappa_{2}, \rho$ and $\zeta$. Now $x^{2} \leq x$ for any $x$ in $\left.] 0,1\right]$ with equality if and only if $x=1$. Since it is not possible for $\sin \alpha, \sin \beta$, and $\sin \gamma$ to equal 1 simultaneously, it follows from (7.13) that

$$
\kappa_{2}<\frac{\sin \alpha+\sin \beta+\sin \gamma}{\sqrt{3} \sin \alpha \sin \beta \sin \gamma}=\frac{2}{\sqrt{3}} \rho .
$$

Now, using both (1.4) and (1.5), we obtain

$$
\begin{aligned}
2 \rho & =\frac{\sin \alpha+\sin \beta+\sin \gamma}{\sin \alpha \sin \beta \sin \gamma} \\
& =\frac{\sin \alpha \sin \beta \sin \gamma(\sin \alpha+\sin \beta+\sin \gamma)^{4}}{\sin ^{2} \alpha \sin ^{2} \beta \sin ^{2} \gamma(\sin \alpha+\sin \beta+\sin \gamma)^{3}} \\
& \leq\left(\frac{\sin \alpha+\sin \beta+\sin \gamma}{3}\right)^{3} \frac{9\left(\sin ^{2} \alpha+\sin ^{2} \beta+\sin ^{2} \gamma\right)^{2}}{\sin ^{2} \alpha \sin ^{2} \beta \sin ^{2} \gamma(\sin \alpha+\sin \beta+\sin \gamma)^{3}} \\
& \leq\left(\frac{\sin ^{2} \alpha+\sin ^{2} \beta+\sin ^{2} \gamma}{\sqrt{3} \sin \alpha \sin \beta \sin \gamma}\right)^{2}=\kappa_{2}^{2} .
\end{aligned}
$$

An application of (1.5) yields the following inequality:

$$
\begin{aligned}
\frac{\zeta}{3 \sqrt{3}} & =\frac{(\sin \alpha+\sin \beta+\sin \gamma)^{2}}{6 \sqrt{3} \sin \alpha \sin \beta \sin \gamma} \\
& \leq \frac{\sin ^{2} \alpha+\sin ^{2} \beta+\sin ^{2} \gamma}{2 \sqrt{3} \sin \alpha \sin \beta \sin \gamma}=\frac{\kappa_{2}}{2} .
\end{aligned}
$$

\section{CONCLUSION}

Of the quality measures that have been investigated, $\iota$ has the disadvantage of being unsymmetric and lacks a stationary point. Among the remaining measures, $\tau$ rejects only needles and $\nu$ rejects only flattened triangles, while the minimal value of $\omega$ selects right triangles. The measures $\kappa_{1}, \kappa_{2}$ and $\kappa_{\infty}$, based on matrix norms, are equivalent to one another and, from the inequalities derived in the final section, we conclude that their behavior is asymptotically equivalent to that of the quality measure $\rho$. Therefore, we can conclude that five classes of triangle quality measures exist whose main properties are summarized in Table 2 . 
TABLE 2. Summary of triangle quality measures: "stat." refers to the existence of a stationary value when the quality is optimal. The kind of "good" triangle is indicated as either right or equilateral.

\begin{tabular}{|c||c|c|c|c|}
\hline & good & stat. & needle & flat. \\
\hline \hline$\frac{1}{2} \rho$ & $\sim 1$ (equil.) & yes & $\gg 1$ & $\gg 1$ \\
\hline$\frac{1}{3 \sqrt{3}} \zeta$ & $\sim 1$ (equil.) & yes & $\gg 1$ & $\gg 1$ \\
\hline$\frac{1}{2} \kappa_{2}$ & $\sim 1$ (equil.) & yes & $\gg 1$ & $\gg 1$ \\
\hline \hline$\frac{1}{2 \sqrt{3}} \iota$ & $\sim 1$ (equil.) & no & $\gg 1$ & $\gg 1$ \\
\hline$\sqrt{3} \frac{R}{|t|_{0}}$ & $\sim 1$ (equil.) & no & $\gg 1$ & $\gg 1$ \\
\hline \hline$\tau$ & $\sim 1$ (equil.) & no & $\gg 1$ & $\sim 2$ \\
\hline \hline$\frac{3 \sqrt{3}}{2} \nu$ & $\sim 1$ (equil.) & yes & $\sim \frac{3 \sqrt{3}}{4}$ & $\gg 1$ \\
\hline \hline $2 \omega$ & $\sim 1$ (right) & yes & $\sim 1$ & $\gg 1$ \\
\hline
\end{tabular}

\section{REFERENCES}

1. T.J. Baker, Element quality in tetrahedral meshes, Proc. 7th Int. Conf. Finite Element Methods in Flow Problems, 1018-1024, Huntsville, U.S.A., 1989.

2. I. BABUŠKA and A.K. AzIZ, On the angle condition in the finite element method, SIAM J. Numer. Anal. 13, 214-227, 1976. MR 56:13700

3. T.J. BAKer, Deformation and quality measures for tetrahedral meshes, Proc. ECCOMAS 2000, Barcelona, Spain, September 2000.

4. R.E. BANK and R.K. Smith, Mesh smoothing using a posteriori error estimates, SIAM J. Numer. Anal. 34, 979-997, 1997. MR 98m:65162

5. P.G. Ciarlet and P.A. Raviart, General Lagrange and Hermite interpolation in $\mathbb{R}^{n}$ with applications to finite element methods, Arch. Rational Mech. Anal. 46, 177-199, 1972. MR 49:1730

6. H.S.M. Coxeter, Introduction to Geometry, Wiley, New York - London, 1961. MR 23:A1251

7. L.A. Freitag and P.M. Knupp, Tetrahedral element shape optimization via the Jacobian determinant and condition number, Proc. 8th Int. Mesh. Roundtable, South Lake Tahoe, U.S.A., October 1999.

8. P.J. Frey and P.L. George, Mesh Generation, Hermes Science Publishing, Oxford \& Paris, 2000. MR 2002c:65001

9. P.M. KnupP, Algebraic mesh quality metrics, SIAM J. Sci. Comput. 23, 193-218, 2001.

10. A. Liu and B. Joe, On the shape of tetrahedra from bisection, Math. Comp. 63 207, 141-154, July 1994. MR 94j:65113

11. V.T. Parthasarathy, C.M. Graichen and A.F. Hathaway, A comparison of tetrahedral quality measures, Fin. Elem. Anal. Des. 15, 255-261, 1993.

12. V.T. RAJAn, Optimality of the Delaunay triangulations in $\mathbb{R}^{d}$, Discrete Comput. Geom. 12, 189-202, 1994. MR 98e:52027

Mechanical and Aerospace Engineering Department, E-Quad, Princeton University, NEW JERSEy 08544

E-mail address: pebay@harrier.princeton.edu

Current E-mail address: pppebay@ca.sandia.gov

Mechanical and Aerospace Engineering Department, E-Quad, Princeton University, NeW JERSEy 08544

E-mail address: baker@tornado.princeton.edu 